\title{
A VARIATIONAL PRINCIPLE FOR THE METRIC MEAN DIMENSION OF FREE SEMIGROUP ACTIONS
}

\author{
MARIA CARVALHO, FAGNER B. RODRIGUES, AND PAULO VARANDAS
}

\begin{abstract}
We consider continuous free semigroup actions generated by a family $\left(g_{y}\right)_{y \in Y}$ of continuous endomorphisms of a compact metric space $(X, d)$, subject to a random walk $\mathbb{P}_{\nu}=\nu^{\mathbb{N}}$ defined on a shift space $Y^{\mathbb{N}}$, where $\left(Y, d_{Y}\right)$ is a compact metric space with finite upper box dimension and $\nu$ is a Borel probability measure on $Y$. With the aim of elucidating the impact of the random walk on the metric mean dimension, we prove a variational principle which relates the metric mean dimension of the semigroup action with the corresponding notions for the associated skew product and the shift map $\sigma$ on $Y^{\mathbb{N}}$, and compare them with the upper box dimension of $Y$. In particular, we obtain exact formulas whenever $\nu$ is homogeneous and has full support. We also discuss several examples to enlighten the roles of the homogeneity, of the support and of the upper box dimension of the measure $\nu$, and to test the scope of our results.
\end{abstract}

\section{INTRODUCTION}

In the late nineties, M. Gromov [9] proposed a new dynamical concept of dimension that was meant to extend the usual topological dimension to broader contexts. This notion, called mean dimension and denoted by mdim, is defined for continuous maps on compact metric spaces in terms of the growth rate of refinements of coverings of the phase space, and is hard to compute in general (cf. [13]). Amid the many virtues of this notion we refer to the following: given compact metric spaces $\left(X_{1}, d_{X_{1}}\right)$ and $\left(X_{2}, d_{X_{2}}\right)$ and continuous maps $T_{1}: X_{1} \rightarrow X_{1}$ and $T_{2}: X_{2} \rightarrow X_{2}$, one has

$$
\operatorname{mdim}\left(X_{1} \times X_{2}, T_{1} \times T_{2}\right) \leqslant \operatorname{mdim}\left(X_{1}, T_{1}\right)+\operatorname{mdim}\left(X_{2}, T_{2}\right) .
$$

Moreover, there are examples where the previous inequality is strict (cf. [13, 18]), which attests how this new concept is subtler than a mere topological dimension. Meanwhile, the topological entropy and its relation to measure-theoretic entropy have become central tools in dynamical systems. The variational principle states that for a continuous dynamical system the supremum of the measure-theoretic entropies of all possible invariant probability measures gives the topological entropy. Yet, one often encounters natural systems with infinite

Date: January 15, 2021.

2010 Mathematics Subject Classification. Primary: 37B05, 37C45, 54F45. Secondary: 37C85, 37D35, $37 \mathrm{~B} 40$

Key words and phrases. Free semigroup action; Skew product; Metric mean dimension; Upper box dimension; Homogeneous measure.

The authors are grateful to the referee for the valuable comments. FR has been financially supported by BREUDS. PV and MC have been supported by CMUP (UID/MAT/00144/2019), which is funded by FCT with national (MCTES) and European structural funds through the programs FEDER, under the partnership agreement PT2020. PV was partially supported by Fundação para a Ciência e Tecnologia (FCT) - Portugal, through the grant CEECIND/03721/2017 of the Stimulus of Scientific Employment, Individual Support 2017 Call. 
entropy, about which the classical theory is less conclusive. E. Lindenstrauss and B. Weiss [13] successfully addressed this problem by introducing a novel entropy-like invariant called metric mean dimension, which we abbreviate into $\operatorname{mim}_{M}$. This concept, inspired by the topological entropy, turns out to be a metric version of Gromov's notion. Besides, it is an upper bound for the mean dimension and, in general, easier to estimate. However, it depends on the metric used, while the topological entropy is metric independent. Despite this drawback, the metric mean dimension exhibits several intrinsic features which makes it a rather compelling notion to be studied. For instance, the topological entropy of a product of dynamics is given by

$$
h_{\mathrm{top}}\left(T_{1} \times T_{2}\right)=h_{\mathrm{top}}\left(T_{1}\right)+h_{\mathrm{top}}\left(T_{2}\right)
$$

and similarly one has

$$
\operatorname{mdim}_{M}\left(X_{1} \times X_{2}, T_{1} \times T_{2}, d_{X_{1}} \times d_{X_{2}}\right)=\operatorname{mdim}_{M}\left(X_{1}, T_{1}, d_{X_{1}}\right)+\operatorname{mdim}_{M}\left(X_{2}, T_{2}, d_{X_{2}}\right) .
$$

Moreover, as happens with the classical variational principle for the topological pressure, Lindenstrauss and Tsukamoto [14] and Tsukamoto [19] established double variational principles relating the mean dimension with potential to the upper and lower rate distortion dimensions, being the former a topological concept and the latter depending both on the metric and the set of invariant probability measures. In this paper, we extend the notion of metric mean dimension to continuous and compactly generated free semigroup actions. (We recall that a topological semigroup is compactly generated if there exists a compact set $K \subset G$ such that $G=\cup_{n \in \mathbb{N}} K^{n}$.)

The research on partially hyperbolic dynamics brought to the stage iterated systems of functions modeling the behavior within the central manifold (see e.g. [3]). This circumstance led to the study of random dynamical systems, and a thorough understanding of the dynamical and ergodic properties of these systems has already been achieved. On the other hand, sequential dynamical systems have been introduced to model physical phenomena: instead of iterating the same dynamics, one allows the system that describes the real events to readjust with time, in a way that matches the inevitable experimental errors. Being a natural approach in the statistical study of lattices (cf. [17]), instead of just a $\mathbb{Z}$ action one considers semigroup actions of dynamical systems. This imparts more comprehensive formulations in statistical mechanics, though it also presents a number of technical problems.

A free semigroup action $\mathbb{S}$ is prescribed by three ingredients: a compact metric space $\left(Y, d_{Y}\right)$, a continuous family $\left(g_{y}: X \rightarrow X\right)_{y \in Y}$ of continuous maps on a compact metric space $(X, d)$, and a random walk $\mathbb{P}$ determined by a Borel probability measure $\nu$ on $Y$ which is invariant by the shift map $\sigma$ acting in $Y^{\mathbb{N}}$. Although an action is not a dynamical system, it is somehow modeled by one, namely the continuous skew product

$$
\begin{aligned}
T_{G}: \quad Y^{\mathbb{N}} \times X & \rightarrow Y^{\mathbb{N}} \times X \\
(\omega, x) & \mapsto\left(\sigma(\omega), g_{\omega_{1}}(x)\right)
\end{aligned}
$$

where $\omega=\left(\omega_{1}, \omega_{2}, \ldots\right)$ is an element of the full unilateral space of sequences $Y^{\mathbb{N}}$. The choice of free semigroup actions is due to two main reasons. Firstly, since we consider only forward iterations it is worthwhile coding the elements in the semigroup by all the finite words associated to the composition of its generators. Secondly, we benefit from the use of a true dynamics given by the skew-product (1.1), an approach which is still not available for general semigroups. We also observe that $\sigma$ is a factor of $T_{G}$ and that, in order to describe the metric mean dimension of a free semigroup action, one is led to address the metric mean dimension 
of skew-products under a randomness constraint $\mathbb{P}$. Now, Ledrappier and Walters' relativized (also known as quenched) variational principle (cf. [12]) asserts that

$$
\sup _{\left\{\mu: \pi_{*} \mu=\mathbb{P}\right\}} h_{\mu}\left(T_{G}\right)=h_{\mathbb{P}}(\sigma)+\int_{Y} h\left(T_{G}, \pi^{-1}(\omega)\right) d \mathbb{P}(\omega)
$$

where $\pi: Y^{\mathbb{N}} \times X \rightarrow Y^{\mathbb{N}}$ is the natural projection and $h\left(T_{G}, \pi^{-1}(\omega)\right)$ is the topological entropy of the skew product dynamics on the fiber $\pi^{-1}(\omega)$. This equality suggests that we look for a similar reformulation of the metric mean dimension of a semigroup action in terms of purely topological ingredients specified by the corresponding skew product and the shift dynamics.

The first difficulty we face in this project is the choice of adequate random walks to drive the action. Let us be more precise. The dimension of a set is roughly the largest dimension of a neighborhood of its points. This is in strong contrast to topological entropy, at least for dynamical systems with local entropy zero, in which case entropy arises associated to the equidistribution of orbits and is determined by the global nature of the dynamics. Moreover, dimensions may be estimated using exterior measures, which give weights according to a geometric structure of the covering elements and do not depend on the locus inside the phase space. This explains why the homogeneous probability measures (that is, those for which the measures of balls with equal radius are uniformly comparable) arise as the most natural ground to approach the metric mean dimension of free semigroup actions. And, in fact, using Borel homogeneous probability measures $\nu$ on $Y$, we establish, under a few mild assumptions, the following variational principle

$$
\overline{\operatorname{mdim}}_{M}\left(Y^{\mathbb{N}} \times X, T_{G}, D \times d\right)=\sup _{\nu}\left(\overline{\operatorname{dim}}_{B}(\operatorname{supp} \nu)+\overline{\operatorname{mdim}}_{M}\left(X, \mathbb{S}, d, \mathbb{P}_{\nu}\right)\right)
$$

which relates the upper metric mean dimension of the skew product with the least upper bound of the sum of the upper box dimension of $\nu$ and the upper metric mean dimension of the semigroup action subject to the random walk $\mathbb{P}_{\nu}=\nu^{\mathbb{N}}$. Besides, we show that when such a random walk $\mathbb{P}_{\nu}$ is built from a homogeneous probability measure $\nu$ with full support, then

$$
\overline{\operatorname{mdim}}_{M}\left(X, \mathbb{S}, d, \mathbb{P}_{\nu}\right)=\overline{\operatorname{mdim}}_{M}\left(Y^{\mathbb{N}} \times X, T_{G}, D \times d\right)-\overline{\operatorname{dim}}_{B} Y
$$

although it may happen that

$$
\sup _{\mu} \overline{\operatorname{mdim}}_{M}\left(X, \mathbb{S}, d, \mathbb{P}_{\mu}\right)>\overline{\operatorname{mdim}}_{M}\left(Y^{\mathbb{N}} \times X, T_{G}, D \times d\right)-\overline{\operatorname{dim}}_{B} Y .
$$

In particular, if the semigroup is finitely generated then $Y$ is a finite set, hence $\overline{\operatorname{dim}}_{B} Y=0$ and the upper metric mean dimensions of the action and the skew-product coincide.

The paper is organized as follows. For the reader's convenience we start with a short glossary with the main definitions we will use. After establishing the aforementioned variational principle, we explore some relevant applications and discuss a number of examples.

\section{Definitions AND PRELIminary RESUlts}

We start recalling the main concepts we use and describing the systems we will work with.

2.1. Metric mean dimension of a map. Let $(X, d)$ be a compact metric space. Given a continuous map $f: X \rightarrow X$ and a non-negative integer $n$, define the dynamical metric $d_{n}: X \times X \rightarrow[0, \infty)$ by

$$
d_{n}(x, z)=\max \left\{d(x, z), d(f(x), f(z)), \ldots, d\left(f^{n}(x), f^{n}(z)\right)\right\}
$$


which generates the same topology as $d$. Having fixed $\varepsilon>0$, we say that a set $E \subset X$ is $(n, \varepsilon)$-separated by $f$ if $d_{n}(x, z)>\varepsilon$ for every $x, z \in E$. In the particular case of $n=1$, we will call such a set $\varepsilon$-separated. Denote by $s(f, n, \varepsilon)$ the maximal cardinality of all $(n, \varepsilon)-$ separated subsets of $X$ by $f$. Due to the compactness of $X$, the number $s(f, n, \varepsilon)$ is finite for every $n \in \mathbb{N}$ and $\varepsilon>0$. We say that $R \subset X$ is a $(n, \varepsilon)$-spanning set if for any $x \in X$ there exists $z \in R$ such that $d_{n}(x, z)<\varepsilon$. When $n=1$, we say that the set is $\varepsilon$-spanning. Let $r(n, \varepsilon)$ be the minimum cardinality of the $(n, \varepsilon)$-spanning subsets of $X$.

Definition 2.1. The lower metric mean dimension of $f$ with respect to the fixed metric $d$ is given by

where

$$
\underline{\operatorname{mim}}_{M}(X, f, d)=\liminf _{\varepsilon \rightarrow 0^{+}} \frac{h(f, \varepsilon)}{|\log \varepsilon|}
$$

$$
h(f, \varepsilon)=\limsup _{n \rightarrow \infty} \frac{1}{n} \log s(f, n, \varepsilon) .
$$

Similarly, the upper metric mean dimension of $f$ with respect to $d$ is the limit

$$
\overline{\operatorname{mdim}}_{M}(X, f, d)=\limsup _{\varepsilon \rightarrow 0^{+}} \frac{h(f, \varepsilon)}{|\log \varepsilon|} .
$$

Clearly, $\underline{\operatorname{mdim}}_{M}(X, f, d)=\overline{\operatorname{mdim}}_{M}(X, f, d)=0$ whenever the topological entropy of $f$, given by $h_{\mathrm{top}}(f)=\lim _{\varepsilon \rightarrow 0^{+}} h(f, \varepsilon)$, is finite.

2.2. Compactly generated semigroup action of continuous maps. Let $(X, d)$ and $\left(Y, d_{Y}\right)$ be compact metric spaces and $\left(g_{y}\right)_{y \in Y}$ be a family of continuous maps $g_{y}: X \rightarrow X$. Denote by $G$ the free semigroup having the set $G_{1}=\left\{g_{y}: y \in Y\right\}$ as generator, where the semigroup operation $\circ$ is the composition of maps. Let $\mathbb{S}$ be the induced free semigroup action

$$
\text { S: } \begin{aligned}
G \times X & \rightarrow X \\
(g, x) & \mapsto g(x)
\end{aligned}
$$

which is said to be compactly generated by $Y$, and denote by $T_{G}$ the associated skew product given by

$$
\begin{array}{rlc}
T_{G}: \quad Y^{\mathbb{N}} \times X & \rightarrow & Y^{\mathbb{N}} \times X \\
(\omega, x) & \mapsto\left(\sigma(\omega), g_{\omega_{1}}(x)\right),
\end{array}
$$

where $\omega=\left(\omega_{1}, \omega_{2}, \ldots\right)$ is an element of the full unilateral space of sequences $Y^{\mathbb{N}}$ and $\sigma$ denotes the shift map acting on $Y^{\mathbb{N}}$. It will be a standing assumption that $T_{G}$ is a continuous map. If for every $n \in \mathbb{N}$ and $\omega=\left(\omega_{1}, \omega_{2}, \ldots\right) \in Y^{\mathbb{N}}$ we write

$$
f_{\omega}^{n}=g_{\omega_{n}} \ldots g_{\omega_{1}}
$$

then

$$
T_{G}^{n}(\omega, x)=\left(\sigma^{n}(\omega), f_{\omega}^{n}(x)\right) .
$$

Consider the set $G_{1}^{*}=G_{1} \backslash\{i d\}$ and, for each $n \in \mathbb{N}$, let $G_{n}^{*}$ denote the space of concatenations of $n$ elements in $G_{1}^{*}$. Similarly, define $G=\bigcup_{n \in \mathbb{N}_{0}} G_{n}$, where $G_{0}=\{i d\}$ and $\underline{g} \in G_{n}$ if and only if $\underline{g}=g_{\omega_{n}} \ldots g_{\omega_{2}} g_{\omega_{1}}$, with $g_{\omega_{j}} \in G_{1}$ (for notational simplicity's sake we will use $g_{j} g_{i}$ instead of the composition $g_{j} \circ g_{i}$ ). In what follows, we will assume that the generator set $G_{1}$ is minimal, meaning that no function $g_{y} \in G_{1}$, for $y \in Y$, can be expressed as a composition of the remaining generators. To summon an element $\underline{g}$ of $G_{n}^{*}$, we will write 
$|\underline{g}|=n$ instead of $\underline{g} \in G_{n}^{*}$. Each element $\underline{g}$ of $G_{n}$ may be seen as a word which originates from the concatenation of $n$ elements in $G_{1}$. Yet, different concatenations may generate the same element in $G$. Nevertheless, in the computations to be done, we shall consider different concatenations instead of the elements in $G$ they create.

2.3. Random walks. A random walk $\mathbb{P}$ on $Y^{\mathbb{N}}$ is a Borel probability measure in this space of sequences which is invariant by the shift map $\sigma$. For instance, we may consider a finite subset $F=\left\{p_{1}, \ldots, p_{k}\right\}$ of $Y$, a probability vector $\left(a_{1}, \cdots, a_{k}\right)$ (that is, a selection of positive real numbers $a_{i}$ such that $\left.\sum_{i=1}^{k} a_{i}=1\right)$, the probability measure $\nu=\sum_{i=1}^{k} a_{i} \delta_{p_{i}}$ on $F$ and the Borel product measure $\mathbb{P}_{\nu}=\nu^{\mathbb{N}}$ on $Y^{\mathbb{N}}$. Such a $\mathbb{P}_{\nu}$ will be called a Bernoulli measure, which is said to be symmetric if $a_{i}=\frac{1}{k}$ for every $i \in\{1, \cdots, k\}$, in which case we denote it by $\mathbb{P}_{k}$. If $Y$ is a Lie group, a natural symmetric random walk is given by $\nu^{\mathbb{N}}$ where $\nu$ is the Haar measure. We denote by $\mathscr{P}\left(Y^{\mathbb{N}}\right)$ the space of Borel probability measures on $Y^{\mathbb{N}}$ and by $\mathscr{P}_{B}\left(Y^{\mathbb{N}}\right)$ its subset of Bernoulli elements. It will be clear later on that the role of each random walk is to point out a particular complex feature of the dynamics, here defined in terms of either the topological entropy (definition in Subsection 2.4) or the metric mean dimension (definition in Subsection 2.5).

2.4. Topological entropy of an action $\mathbb{S}$. Given $\varepsilon>0$ and $\underline{g}:=g_{\omega_{n}} \ldots g_{\omega_{2}} g_{\omega_{1}} \in G_{n}$, the $n$ th-dynamical ball $B_{n}(x, \underline{g}, \varepsilon)$ is the set

$$
B_{n}(x, \underline{g}, \varepsilon):=\left\{z \in X: d\left(\underline{g}_{j}(z), \underline{g}_{j}(x)\right) \leqslant \varepsilon, \forall 0 \leqslant j \leqslant n\right\}
$$

where, for every $0 \leqslant j \leqslant n$, the notation $\underline{g}_{j}$ stands for the concatenation $g_{\omega_{j}} \ldots g_{\omega_{2}} g_{\omega_{1}}$ in $G_{j}$, and $\underline{g}_{0}=i d$. Observe that this is a classical ball with respect to the dynamical metric $d_{\underline{g}}$ defined by

$$
d_{\underline{g}}(x, z):=\max _{0 \leqslant j \leqslant n} d\left(\underline{g}_{j}(x), \underline{g}_{j}(z)\right) .
$$

Notice also that both the dynamical ball and the dynamical metric depend on the underlying concatenation of generators $g_{\omega_{n}} \ldots g_{\omega_{1}}$ and not on the semigroup element $\underline{g}$, since the latter may have distinct representations.

Given $\underline{g}=g_{\omega_{n}} \ldots g_{\omega_{1}} \in G_{n}$, we say that a set $K \subset X$ is $(\underline{g}, n, \varepsilon)$-separated if $d_{g}(x, z)>\varepsilon$ for any two distinct elements $x, z \in K$. The largest cardinality of any $(\underline{g}, n, \varepsilon)$-separated subset on $X$ is denoted by $s(\underline{g}, n, \varepsilon)$ (or, equivalently, $s\left(g_{\omega_{n}} \ldots g_{\omega_{1}}, n, \varepsilon\right)$ ). A set $K \subset X$ is said to be $(\underline{g}, n, \varepsilon)$-spanning if for every $x \in X$ there is $k \in K$ such that $d_{g}(x, k) \leqslant \varepsilon$. The smallest cardinality of any $(\underline{g}, n, \varepsilon)$-spanning subset on $X$ is denoted by $\underline{r}(\underline{g}, n, \varepsilon)$ (or $\left.r\left(g_{\omega_{n}} \ldots g_{\omega_{1}}, n, \varepsilon\right)\right)$.

Definition 2.2. The topological entropy of the semigroup action $\mathbb{S}$ with respect to a fixed set of generators $G_{1}$ and a random walk $\mathbb{P}$ in $Y^{\mathbb{N}}$ is given by

$$
h_{\mathrm{top}}(\mathbb{S}, \mathbb{P}):=\lim _{\varepsilon \rightarrow 0^{+}} \limsup _{n \rightarrow \infty} \frac{1}{n} \log \int_{Y^{\mathbb{N}}} s\left(g_{\omega_{n}} \ldots g_{\omega_{1}}, n, \varepsilon\right) d \mathbb{P}(\omega)
$$

where $\omega=\omega_{1} \omega_{2} \cdots \omega_{n} \cdots$. The topological entropy of the semigroup action $\mathbb{S}$ is then defined by

$$
h_{\text {top }}(\mathbb{S})=\sup _{\mathbb{P}} h_{\text {top }}(\mathbb{S}, \mathbb{P})
$$


We observe that the semigroup may have multiple generating sets, and the dynamical or ergodic properties (as the topological entropy) depend on the chosen generator set. More information regarding these concepts in the case of finitely generated free semigroup actions may be read in $[5,6,16]$.

2.5. Metric mean dimension of an action $\mathbb{S}$. Let $(X, d)$ be a compact metric space and $\mathbb{S}$ be the free semigroup action induced on $(X, d)$ by a family of continuous maps $\left(g_{y}: X \rightarrow\right.$ $X)_{y \in Y}$.

Definition 2.3. The upper and lower metric mean dimension of the free semigroup action $\mathbb{S}$ on $(X, d)$ with respect to a fixed set of generators $G_{1}$ and a random walk $\mathbb{P}$ in $Y^{\mathbb{N}}$ are given respectively by

$$
\begin{aligned}
\overline{\operatorname{mdim}}_{M}(X, \mathbb{S}, d, \mathbb{P}) & =\limsup _{\varepsilon \rightarrow 0^{+}} \frac{h(X, \mathbb{S}, \mathbb{P}, \varepsilon)}{-\log \varepsilon} \\
\underline{\operatorname{mim}}_{M}(X, \mathbb{S}, d, \mathbb{P}) & =\liminf _{\varepsilon \rightarrow 0^{+}} \frac{h(X, \mathbb{S}, \mathbb{P}, \varepsilon)}{-\log \varepsilon}
\end{aligned}
$$

where

$$
h(X, \mathbb{S}, \mathbb{P}, \varepsilon)=\limsup _{n \rightarrow \infty} \frac{1}{n} \log \int_{Y^{\mathbb{N}}} s\left(g_{\omega_{n}} \ldots g_{\omega_{1}}, n, \varepsilon\right) d \mathbb{P}(\omega)
$$

2.6. Upper box dimension. Let $\left(Y, d_{Y}\right)$ be a compact metric space.

Definition 2.4. The upper box dimension of $\left(Y, d_{Y}\right)$ is given by

$$
\overline{\operatorname{dim}}_{B} Y=\limsup _{\varepsilon \rightarrow 0^{+}} \frac{\log N(\varepsilon)}{|\log \varepsilon|}
$$

where $N(\varepsilon)$ stands for the maximal cardinality of an $\varepsilon$-separated set in $\left(Y, d_{Y}\right)$.

Consider now a Borel probability measure $\nu$ on $Y$.

Definition 2.5. The upper box dimension of $\nu$ is given by

$$
\overline{\operatorname{dim}}_{B} \nu=\lim _{\delta \rightarrow 0^{+}} \inf \left\{\overline{\operatorname{dim}}_{B} Z: Z \subset Y \quad \text { and } \nu(Z) \geqslant 1-\delta\right\} .
$$

It is worth mentioning that, although the upper box dimension of a set $Z$ coincides with the upper box dimension of its closure, the upper box dimension of a probability measure is intended to estimate the size of subsets rather than the entire support of the measure (that is, the smallest closed subset with full measure). Indeed, it may happen that $\overline{\operatorname{dim}}_{B} \nu<$ $\overline{\operatorname{dim}}_{B}(\operatorname{supp} \nu)(\mathrm{cf}$. Example 7.1 in [15]). We refer the reader to $[10,15]$ for excellent accounts on dimension theory.

2.7. Homogeneous measures. Let $\nu$ be a Borel probability measure on the compact metric space $\left(Y, d_{Y}\right)$. A balanced measure should give the same probability to any two balls with the same radius, but this is in general a too strong demanding. Instead, we weaken the request in the following way.

Definition 2.6. We say that $\nu$ is homogeneous if there exists $L>0$ such that

$$
\nu\left(B\left(y_{1}, 2 \varepsilon\right)\right) \leqslant L \nu\left(B\left(y_{2}, \varepsilon\right)\right) \quad \forall y_{1}, y_{2} \in \operatorname{supp} \nu \quad \forall \varepsilon>0 .
$$


For instance, the Lebesgue measure on $[0,1]$, atomic measures and probability measures absolutely continuous with respect to the latter ones, with densities bounded away from zero and infinity, are examples of homogeneous probability measures. We denote by $\mathcal{H}_{Y}$ the set of such homogeneous Borel probability measures on $Y$.

By definition, every homogeneous measure satisfies

$$
\nu(B(y, 2 \varepsilon)) \leqslant L \nu(B(y, \varepsilon)) \quad \forall y \in \operatorname{supp} \nu \quad \forall \varepsilon>0
$$

and, as $\nu\left(B\left(y_{1}, \varepsilon\right)\right) \leqslant \nu\left(B\left(y_{1}, 2 \varepsilon\right)\right)$,

$$
\nu\left(B\left(y_{1}, \varepsilon\right)\right) \leqslant L \nu\left(B\left(y_{2}, \varepsilon\right)\right) \quad \forall y_{1}, y_{2} \in \operatorname{supp} \nu \quad \forall \varepsilon>0 .
$$

A measure $\nu$ satisfying (2.6) is said to be a doubling measure. Although the two concepts (2.6) and (2.7) are unrelated in general, if $Y$ is a subset of an Euclidean space $\mathbb{R}^{k}$ then any probability $\nu$ satisfying (2.7) is a doubling measure. Indeed, as there is a constant $C_{k}$ such that $\operatorname{Leb}(B(y, r))=C_{k} r^{k}$ for every $y \in Y$ and every $r>0$, any ball $B(y, 2 \varepsilon)$ can be covered by at most $2^{k}$ balls of radius $\varepsilon$; we now apply (2.5). For a discussion on conditions on $Y$ which ensure the existence of homogeneous measures and further relations between homogeneity and the doubling property we refer the reader to [2, Section 4] and references therein.

\section{MAin RESUlts}

Given a compact metric space $\left(Y, d_{Y}\right)$, consider on $Y^{\mathbb{N}}$ the metric

$$
D\left(\left(y_{n}\right)_{n \in \mathbb{N}},\left(z_{n}\right)_{n \in \mathbb{N}}\right)=\sum_{i=1}^{\infty} \frac{d_{Y}\left(y_{i}, z_{i}\right)}{2^{i}} .
$$

It is known (cf. [20, Theorem 5]) that

$$
\overline{\operatorname{mdim}}\left(Y^{\mathbb{N}}, \sigma, D\right)=\overline{\operatorname{dim}}_{B} Y
$$

where $\sigma: Y^{\mathbb{N}} \rightarrow Y^{\mathbb{N}}$ is the shift map. Denote by $\mathscr{P}_{P}\left(Y^{\mathbb{N}}\right)$ the set of product probability measures $\mathbb{P}_{\nu}=\nu^{\mathbb{N}}$ on $Y^{\mathbb{N}}$, where $\nu$ is any Borel probability measure on $Y$.

Let $(X, d)$ be a compact metric space and take on $Y^{\mathbb{N}} \times X$ the product metric $D \times d$. Given a family of continuous endomorphisms $\left(g_{y}: X \rightarrow X\right)_{y \in Y}$, consider the free semigroup action $\mathbb{S}$ induced on $(X, d)$ by $G=\left\{g_{y}: y \in Y\right\}$ and a random walk $\mathbb{P}_{\nu} \in \mathscr{P}_{P}\left(Y^{\mathbb{N}}\right)$. Take its associated skew product $T_{G}: Y^{\mathbb{N}} \times X \rightarrow Y^{\mathbb{N}} \times X$, with the metric $D \times d$. We start relating the metric mean dimensions of $T_{G}$ and $\mathbb{S}$ with the upper box dimension of the support of the measure $\nu$.

Theorem A. If $\overline{\operatorname{dim}}_{B} Y<+\infty$ and $\nu \in \mathcal{H}_{Y}$, then:

(a) $\overline{\operatorname{dim}}_{B}(\operatorname{supp} \nu)+\overline{\operatorname{mdim}}_{M}\left(X, \mathbb{S}, d, \mathbb{P}_{\nu}\right) \leqslant \overline{\operatorname{mim}}_{M}\left(Y^{\mathbb{N}} \times X, T_{G}, D \times d\right)$.

(b) If, in addition, $\operatorname{supp} \nu=Y$,

$$
\overline{\operatorname{dim}}_{B} Y+\overline{\operatorname{mdim}}_{M}\left(X, \mathbb{S}, d, \mathbb{P}_{\nu}\right)=\overline{m \operatorname{mim}}_{M}\left(Y^{\mathbb{N}} \times X, T_{G}, D \times d\right) .
$$

Observe that if item (b) of Theorem A is rewritten as

$$
\overline{\operatorname{mdim}}_{M}\left(X, \mathbb{S}, d, \mathbb{P}_{\nu}\right)=\overline{\operatorname{mdim}}_{M}\left(Y^{\mathbb{N}} \times X, T_{G}, D \times d\right)-\overline{\operatorname{dim}}_{B} Y
$$


then the right-hand side deals only with geometrical objects, which are independent of the random walk $\mathbb{P}_{\nu}$. The previous equality extends to the notion of metric mean dimension the formula of Bufetov

$$
h_{\mathrm{top}}\left(\mathbb{S}, \mathbb{P}_{p}\right)=h_{\mathrm{top}}\left(T_{G}\right)-h_{\mathrm{top}}(\sigma)
$$

regarding the topological entropy of a finitely generated free semigroup action with respect to the symmetric Bernoulli random walk $\mathbb{P}_{p}$ (cf. $[4,7]$ ). In particular, this raises the question of whether

$$
\sup _{\nu \in \mathcal{H}_{Y}} \overline{\operatorname{mdim}}_{M}\left(X, \mathbb{S}, d, \mathbb{P}_{\nu}\right)=\overline{\operatorname{mdim}}_{M}\left(Y^{\mathbb{N}} \times X, T_{G}, D \times d\right)-\overline{\operatorname{dim}}_{B} Y .
$$

Such a variational principle turns out to be false (cf. Example 6.3.1 on Section 6).

Notice also that taking homogeneous measures is a fundamental requirement in Theorem A, and that the equality (3.1) may be realized by homogeneous measures that do not maximize the upper box dimension. For a discussion about the demands on the measure $\nu$, we refer the reader to Section 6.

We do not know a complete characterization of the compact metric spaces $Y$ for which a Borel homogeneous probability measure with full support exists. This is relevant since, for those spaces, one has:

Corollary I. If $\overline{\operatorname{dim}}_{B} Y<+\infty$ and there exists a Borel homogeneous probability measure on $Y$ with full support, then

$$
\sup _{\nu \in \mathcal{H}_{Y}}\left\{\overline{\operatorname{dim}}_{B}(\operatorname{supp} \nu)+\overline{\operatorname{mdim}}_{M}\left(X, \mathbb{S}, d, \mathbb{P}_{\nu}\right)\right\}=\overline{\operatorname{mim}}_{M}\left(Y^{\mathbb{N}} \times X, T_{G}, D \times d\right) .
$$

Moreover, the supremum is attained at every $\nu_{0} \in \mathcal{H}_{Y}$ such that $\operatorname{supp} \nu_{0}=Y$.

We may ask whether the metric mean dimension of a free semigroup action is the average of the metric mean dimensions of the generators. From Theorem A we deduce the following relation between these two values whenever the space $Y$ has a Borel homogeneous probability measure with full support.

Corollary II. If $\overline{\operatorname{dim}}_{B} Y<+\infty$, then:

(a) For every $\nu_{0} \in \mathcal{H}_{Y}$ such that $\operatorname{supp} \nu_{0}=Y$,

$$
\overline{\operatorname{mdim}}_{M}\left(X, \mathbb{S}, d, \mathbb{P}_{\nu_{0}}\right) \geqslant \sup _{y \in Y}\left\{\overline{\operatorname{mim}}_{M}\left(X, g_{y}, d\right)\right\}-\overline{\operatorname{dim}}_{B} Y .
$$

(b) For every $\eta \in \mathscr{P}(Y)$ and any $\nu_{0} \in \mathcal{H}_{Y}$ such that $\operatorname{supp} \nu_{0}=Y$,

$$
\overline{\operatorname{mdim}}_{M}\left(X, \mathbb{S}, d, \mathbb{P}_{\nu_{0}}\right) \geqslant \int_{Y} \overline{\operatorname{mdim}}_{M}\left(X, g_{y}, d\right) d \eta(y)-\overline{\operatorname{dim}}_{B} Y .
$$

(c) In particular, when $\overline{\operatorname{dim}}_{B} Y=0$, for every $\nu_{0} \in \mathcal{H}_{Y}$ such that $\operatorname{supp} \nu_{0}=Y$

$$
\overline{\operatorname{mdim}}_{M}\left(X, \mathbb{S}, d, \mathbb{P}_{\nu_{0}}\right) \geqslant \int_{Y} \overline{\operatorname{mdim}}_{M}\left(X, g_{y}, d\right) d \nu_{0}(y)
$$

and the equality cannot be attained unless we have for $\nu_{0}$-almost every $y \in Y$

$$
\overline{\operatorname{mdim}}_{M}\left(X, g_{y}, d\right)=\sup _{\alpha \in Y}\left\{\overline{\operatorname{mdim}}_{M}\left(X, g_{\alpha}, d\right)\right\} .
$$


Let us apply Theorem A to two relevant particular cases. Firstly assume that $Y$ is finite. In this case, $\overline{\operatorname{dim}}_{B} Y=0$, and every probability $\nu$ on $Y$ is homogeneous, satisfying also $\overline{\operatorname{dim}}_{B}(\operatorname{supp} \nu)=\overline{\operatorname{dim}}_{B} Y$.

Corollary III. Assume that $Y=\{1,2, \ldots, p\}$ for some $p \in \mathbb{N}$. If $\nu$ is a Borel probability measure on $Y$ with full support, then

$$
\overline{\operatorname{mdim}}_{M}\left(X, \mathbb{S}, d, \mathbb{P}_{\nu}\right)=\overline{\operatorname{mdim}}_{M}\left(Y^{\mathbb{N}} \times X, T_{G}, D \times d\right) .
$$

In what follows, we abbreviate into Leb the Lebesgue measure on $[0,1]^{k}, k \in \mathbb{N}$. Consider the Euclidean metric $d$ in $[0,1]^{k}$ and the probability $\mathbb{P}_{\text {Leb }}:=\operatorname{Leb}^{\mathbb{N}}$ on $\left([0,1]^{k}\right)^{\mathbb{N}}$. The next result is an immediate consequence of item (b) of Theorem A and the equality (cf. [13, 20])

$$
\overline{\operatorname{mdim}}_{M}\left(\left([0,1]^{k}\right)^{\mathbb{N}}, \sigma, D\right)=\overline{\operatorname{dim}}_{B}\left([0,1]^{k}\right) .
$$

Corollary IV. Suppose that $Y=[0,1]^{k}$ for some $k \in \mathbb{N}$. Then

$$
\overline{\operatorname{mdim}}_{M}\left(X, \mathbb{S}, d, \mathbb{P}_{L e b}\right)=\overline{\operatorname{mdim}}_{M}\left(\left([0,1]^{k}\right)^{\mathbb{N}} \times X, T_{G}, D \times d\right)-k .
$$

\section{Proof of Theorem A}

Fix an arbitrary $\varepsilon>0$, and take $K=K(\varepsilon) \geqslant 1$ such that $\sum_{i>K}^{\infty} \frac{\operatorname{diam}(Y)}{2^{i}}<\frac{\varepsilon}{2}$. For $\nu \in \mathcal{H}_{Y}$, consider $Z=\operatorname{supp} \nu$. Afterwards, choose $E \subset Z$ a maximal $\frac{\varepsilon}{4}$-separated subset and denote by $N_{Z}(\varepsilon)$ its cardinality. By the definition of upper box dimension, we have

$$
\overline{\operatorname{dim}}_{B} Z=\limsup _{\varepsilon \rightarrow 0^{+}} \frac{N_{Z}(\varepsilon)}{-\log \left(\frac{\varepsilon}{4}\right)}=\limsup _{\varepsilon \rightarrow 0^{+}} \frac{N_{Z}(\varepsilon)}{-\log \varepsilon} .
$$

For each $n \in \mathbb{N}$ and each point $\left(p_{i_{1}}, \ldots, p_{i_{n+K}}\right) \in E^{n+K}$, consider the cylinder

$$
C_{i_{1}, i_{2}, \ldots, i_{n+K}}=\left\{\omega \in Y^{\mathbb{N}}: \omega_{0} \in B\left(p_{i_{1}}, \frac{\varepsilon}{4}\right), \ldots, \omega_{n-1} \in B\left(p_{i_{n+K}}, \frac{\varepsilon}{4}\right)\right\}
$$

and notice that every $\omega, \theta \in C_{i_{1}, i_{2}, \ldots, i_{n+K}}$ satisfy $D_{n}(\omega, \theta)<\varepsilon$. Indeed, since $d_{Y}\left(\omega_{i}, \theta_{i}\right)<\frac{\varepsilon}{2}$ for every $0 \leqslant i \leqslant n+K-1$, then

$$
D\left(\sigma^{j}(\omega), \sigma^{j}(\theta)\right) \leqslant \sum_{i=1}^{n+K-j} \frac{\varepsilon}{2^{i+1}}+\sum_{i>n+K-j} \frac{\operatorname{diam}(Y)}{2^{i}}<\varepsilon \quad \forall 0 \leqslant j \leqslant n .
$$

Since $E \subset Z$ is a maximal $\frac{\varepsilon}{4}$-separated subset of $Z$, it is an $\frac{\varepsilon}{4}$-spanning set of $Z$; hence the collection defined by (4.1) is a covering of $Z^{\mathbb{N}}$. Moreover, if we associate to each $\underline{p}=$ $\left(p_{i_{0}}, \ldots, p_{i_{n+K-1}}\right) \in E^{n+K}$ a point $\omega^{(\underline{p})} \in C_{i_{1}, i_{2}, \ldots, i_{n+K}}$ so that its $j$ th-coordinate $\omega_{j}^{(\underline{p})}$ coincides with $p_{i_{j}}$ for every $1 \leqslant j \leqslant n+K$, then we obtain a $(\sigma, n+K, \varepsilon / 4)$-separated subset of $Y^{\mathbb{N}}$. In fact, if $\omega^{(\underline{p})} \neq \omega^{(\underline{q})}$ then there exists $1 \leqslant j \leqslant n+K$ such that their $j$-coordinates $\omega_{j}^{(\underline{p})}$ and $\omega_{j}^{(q)}$ belong to $E$ and are different, and so

$$
D\left(\sigma^{j}\left(\omega^{(\underline{p})}\right), \sigma^{j}\left(\omega^{(\underline{q})}\right)\right) \geqslant \min _{y_{1} \neq y_{2} \in E} d_{Y}\left(y_{1}, y_{2}\right) \geqslant \frac{\varepsilon}{4} .
$$


Consequently,

$$
\begin{aligned}
\int_{Y^{\mathbb{N}}} s\left(g_{\omega}, n, \frac{\varepsilon}{4}\right) d \mathbb{P}_{\nu}(\omega) & =\int_{Z^{\mathbb{N}}} s\left(g_{\omega}, n, \frac{\varepsilon}{4}\right) d \mathbb{P}_{\nu}(\omega) \\
& \leqslant \sum_{\underline{i}=\left(i_{1}, i_{2}, \ldots, i_{n+K}\right)}\left[\max _{\omega \in C_{\underline{i}}} s\left(g_{\omega}, n, \frac{\varepsilon}{4}\right) \times \mathbb{P}_{\nu}\left(C_{\underline{i}} \cap Z^{\mathbb{N}}\right)\right] .
\end{aligned}
$$

We observe now that the image of the map $s\left(\cdot, n, \frac{\varepsilon}{4}\right): C_{\underline{i}} \rightarrow \mathbb{Z}$ defined by $\omega \in C_{\underline{i}} \mapsto s\left(g_{\omega}, n, \frac{\varepsilon}{4}\right)$ is contained in $\left[0, s\left(T_{G}, n, \frac{\varepsilon}{4}\right)\right] \cap \mathbb{Z}$. So it has a maximum in $\mathbb{Z}$, which ensures that $\omega^{(i)}$ can be chosen as a point in $C_{\underline{i}}$ where the $\max _{C_{\underline{i}}} s\left(g_{\omega}, n, \frac{\varepsilon}{4}\right)$ is attained. Combining this information with (4.2), the fact that $\mathbb{P}_{\nu}$ is a product measure and the homogeneity assumption on $\nu$, we obtain

$$
\begin{aligned}
\int_{Z^{\mathbb{N}}} s\left(g_{\omega}, n, \frac{\varepsilon}{4}\right) d \mathbb{P}_{\nu}(\omega) & \leqslant\left[\sum_{\underline{i}} \max _{\omega \in C_{\underline{i}}} s\left(g_{\omega}, n, \frac{\varepsilon}{4}\right)\right] \times \max _{\underline{i}} \mathbb{P}_{\nu}\left(C_{\underline{i}} \cap Z^{\mathbb{N}}\right) \\
& \leqslant\left[\sum_{\underline{i}} s\left(g_{\omega(\underline{i})}, n, \frac{\varepsilon}{4}\right)\right] \times \max _{\underline{i}} \prod_{j=0}^{n+K-1} \nu\left(B\left(p_{i_{j}}, \frac{\varepsilon}{4}\right)\right) \\
& \leqslant s\left(T_{G}, n, \frac{\varepsilon}{4}\right)\left(\frac{1}{N_{Z}(\varepsilon)}\right)^{n+K} .
\end{aligned}
$$

The inequality

$$
\left[\sum_{\underline{i}} s\left(g_{\omega(\underline{i})}, n, \frac{\varepsilon}{4}\right)\right] \leqslant s\left(T_{G}, n, \frac{\varepsilon}{4}\right)
$$

is a consequence of the fact that, if $\left\{x_{1}^{(i)}, \ldots, x_{s\left(g_{\omega^{(i)}}, n, \varepsilon\right)}\right\}$ is a $\left(g_{\omega^{(i)}}, n, \frac{\varepsilon}{4}\right)$-separated set of biggest cardinality, then

$$
\bigcup_{\underline{i}}\left\{\left(\omega^{(\underline{i})}, x_{1}^{(\underline{i})}\right), \ldots,\left(\omega^{(\underline{i})}, x_{s\left(g_{\omega}^{(i)}, n, \frac{\varepsilon}{4}\right)}^{(i)}\right)\right\}
$$

is a $\left(T_{G}, n, \frac{\varepsilon}{4}\right)$-separated set. On the other hand, the inequality

$$
\max _{\underline{i}} \prod_{j=0}^{n+K-1} \nu\left(B\left(p_{i_{j}}, \frac{\varepsilon}{4}\right)\right) \leqslant\left(\frac{1}{N_{Z}(\varepsilon)}\right)^{n+K}
$$

is due to the homogeneity of $\nu$. In fact, for every $q \in \operatorname{supp} \nu$, any $p_{i_{j}}$ and all $\underline{i}$, one has

$$
\nu\left(B\left(p_{i_{j}}, \frac{\varepsilon}{8}\right)\right) \leqslant L \nu\left(B\left(q, \frac{\varepsilon}{8}\right)\right) \quad \forall \varepsilon>0
$$

and, as the balls in the collection $\left(B\left(e, \frac{\varepsilon}{8}\right)\right)_{e \in E}$ are pairwise disjoint (due to the maximality of $E$ as an $\frac{\varepsilon}{4}$-separated set),

$$
1 \geqslant \nu\left(\bigcup_{e \in E} B\left(e, \frac{\varepsilon}{8}\right)\right)=\sum_{e \in E} \nu\left(B\left(e, \frac{\varepsilon}{8}\right)\right) \geqslant N_{Z}(\varepsilon) L \nu\left(B\left(q, \frac{\varepsilon}{8}\right)\right) .
$$

Thus

$$
\nu\left(B\left(q, \frac{\varepsilon}{8}\right)\right) \leqslant \frac{1}{L} \frac{1}{N_{Z}(\varepsilon)} \quad \forall q \in \operatorname{supp} \nu
$$


Finally, since $\nu$ is a doubling measure, there exists $L>0$ (independent of $\varepsilon$ ) such that

$$
\nu\left(B\left(q, \frac{\varepsilon}{4}\right)\right) \leqslant L \nu\left(B\left(q, \frac{\varepsilon}{8}\right)\right) \quad \forall q \in \operatorname{supp} \nu .
$$

We may now complete the proof of Theorem A (a):

$$
\begin{aligned}
\overline{\operatorname{mdim}}_{M}\left(X, \mathbb{S}, d, \mathbb{P}_{\nu}\right) & =\limsup _{\varepsilon \rightarrow 0^{+}} \frac{\limsup _{n \rightarrow \infty} \frac{1}{n} \log \int_{Z^{\mathbb{N}}} s\left(g_{\omega}, n, \frac{\varepsilon}{4}\right) d \mathbb{P}_{\nu}(\omega)}{-\log \frac{\varepsilon}{4}} \\
& \leqslant \overline{\operatorname{mim}}_{M}\left(Y^{\mathbb{N}} \times X, T_{G}, D \times d\right)-\limsup \frac{\log N_{Z}(\varepsilon)}{-\log \varepsilon} \\
& =\overline{\operatorname{mim}}_{M}\left(Y^{\mathbb{N}} \times X, T_{G}, D \times d\right)-\overline{\operatorname{dim}}_{B} Z \\
& =\overline{\operatorname{mim}}_{M}\left(Y^{\mathbb{N}} \times X, T_{G}, D \times d\right)-\overline{\operatorname{dim}}_{B}(\operatorname{supp} \nu) .
\end{aligned}
$$

Before proving item (b) of Theorem A, we establish an alternative characterization of the metric mean dimension using spanning sets.

Lemma 4.1. If $\mathbb{S}$ is a free semigroup action on $(X, d)$ with respect to a random walk $\mathbb{P}$ in $Y^{\mathbb{N}}$ then

$$
\overline{\operatorname{mdim}}_{M}(X, \mathbb{S}, d, \mathbb{P})=\limsup _{\varepsilon \rightarrow 0^{+}} \frac{h^{r}(X, \mathbb{S}, \mathbb{P}, \varepsilon)}{-\log \varepsilon}
$$

where

$$
h^{r}(X, \mathbb{S}, \mathbb{P}, \varepsilon)=\limsup _{n \rightarrow \infty} \frac{1}{n} \log \int_{Y^{\mathbb{N}}} r\left(g_{\omega_{n}} \ldots g_{\omega_{1}}, n, \varepsilon\right) d \mathbb{P}(\omega)
$$

and $r\left(g_{\omega_{n}} \ldots g_{\omega_{1}}, n, \varepsilon\right)$ stands for the minimal cardinal of an $(n, \varepsilon)$-spanning set in $(X, d)$.

Proof. In view of Definition 2.3 it is enough to prove that

$$
\limsup _{\varepsilon \rightarrow 0^{+}} \frac{h(X, \mathbb{S}, \mathbb{P}, \varepsilon)}{-\log \varepsilon}=\limsup _{\varepsilon \rightarrow 0^{+}} \frac{h^{r}(X, \mathbb{S}, \mathbb{P}, \varepsilon)}{-\log \varepsilon}
$$

where $h(X, \mathbb{S}, \mathbb{P}, \varepsilon)$ is given by $(2.3)$. In fact, since $r\left(g_{\omega_{n}} \ldots g_{\omega_{1}}, n, \varepsilon\right) \leqslant s\left(g_{\omega_{n}} \ldots g_{\omega_{1}}, n, \varepsilon\right) \leqslant$ $r\left(g_{\omega_{n}} \ldots g_{\omega_{1}}, n, \frac{\varepsilon}{2}\right)$ for every $n \in \mathbb{N}, \varepsilon>0$ and $\mathbb{P}$-a.e. $\omega$ (the arguments are identical to the ones with a single continuous map), it is clear that

$$
h^{r}(X, \mathbb{S}, \mathbb{P}, \varepsilon) \leqslant h(X, \mathbb{S}, \mathbb{P}, \varepsilon) \leqslant h^{r}\left(X, \mathbb{S}, \mathbb{P}, \frac{\varepsilon}{2}\right)
$$

for every $\varepsilon>0$. This ends the proof of the lemma.

We now resume the proof of Theorem A (b). Although we are interested in homogeneous measures with full support, the next result is more general.

Proposition 4.2. If $\nu \in \mathcal{H}_{Y}$ then

$$
\overline{\operatorname{dim}}_{B}(\operatorname{supp} \nu)+\overline{\operatorname{mdim}}_{M}\left(X, \mathbb{S}, d, \mathbb{P}_{\nu}\right)=\overline{\operatorname{mdim}}_{M}\left((\operatorname{supp} \nu)^{\mathbb{N}} \times X, T_{G}, D \times d\right) .
$$


Proof. We start remarking that the argument to prove Theorem A (a) also shows that if $\nu \in \mathcal{H}_{Y}$ then

$$
\overline{\operatorname{dim}}_{B}(\operatorname{supp} \nu)+\overline{\operatorname{mdim}}_{M}\left(X, \mathbb{S}, d, \mathbb{P}_{\nu}\right) \leqslant \overline{\operatorname{mdim}}_{M}\left((\operatorname{supp} \nu)^{\mathbb{N}} \times X, T_{G}, D \times d\right) .
$$

We are left to establish the reverse inequality.

Fix an arbitrary $\varepsilon>0$ and consider an integer $K=K(\varepsilon) \geqslant 1$ such that $\sum_{i>K}^{\infty} \frac{\operatorname{diam}(Y)}{2^{i}}<\frac{\varepsilon}{2}$. For $\nu \in \mathcal{H}_{Y}$, take $Z=\operatorname{supp} \nu$ and choose a maximal $\frac{\varepsilon}{4}$-separated set $E \subset Z$, whose cardinality is denoted by $N_{Z}(\varepsilon)$. By the definition of upper box dimension, $\lim \sup _{\varepsilon \rightarrow 0^{+}} \frac{N_{Z}(\varepsilon)}{-\log \varepsilon}=\overline{\operatorname{dim}}_{B} Z$. For each $n \in \mathbb{N}$ and each point $\left(p_{i_{1}}, \ldots, p_{i_{n+K}}\right) \in E^{n+K}$, consider the cylinder

$$
C_{i_{1}, i_{2}, \ldots, i_{n+K}}=\left\{\omega \in Y^{\mathbb{N}}: \omega_{1} \in B\left(p_{i_{1}}, \varepsilon / 4\right), \ldots, \omega_{n} \in B\left(p_{i_{n+K}}, \varepsilon / 4\right)\right\}
$$

and notice that every $\omega, \theta \in C_{i_{1}, i_{2}, \ldots, i_{n+K}}$ satisfy $D_{n}(\omega, \theta)<\varepsilon$. Indeed, since $d_{Y}\left(\omega_{i}, \theta_{i}\right)<\frac{\varepsilon}{2}$ for every $1 \leqslant i \leqslant n+K$, then

$$
D\left(\sigma^{j}(\omega), \sigma^{j}(\theta)\right) \leqslant \sum_{i=1}^{n+K-j} \frac{\varepsilon}{2^{i+1}}+\sum_{i>n+K-j} \frac{\operatorname{diam}(Y)}{2^{i}}<\varepsilon \quad \forall 0 \leqslant j \leqslant n .
$$

Since $E \subset Z$ is a maximal $\frac{\varepsilon}{4}$-separated subset of $Z$, it is an $\frac{\varepsilon}{4}$-spanning set of $Z$; hence the collection defined by $(4.3)$ is a covering of $Z^{\mathbb{N}}$. Moreover, if we select for each $\underline{p}=$ $\left(p_{i_{1}}, \ldots, p_{i_{n+K}}\right) \in E^{n+K}$ a point $\omega^{(\underline{p})} \in C_{i_{1}, i_{2}, \ldots, i_{n+K}}$ so that its $j$ th-coordinate $\omega_{j}^{(\underline{p})}$ coincides with $p_{i_{j}}$ for every $1 \leqslant j \leqslant n+K$, then we obtain a $(\sigma, n, \varepsilon)$-spanning subset of $Z^{\mathbb{N}}$. Consequently,

$$
\begin{aligned}
\int_{Y^{\mathbb{N}}} r\left(g_{\omega}, n, \varepsilon\right) d \mathbb{P}_{\nu}(\omega) & =\int_{Z^{\mathbb{N}}} r\left(g_{\omega}, n, \varepsilon\right) d \mathbb{P}_{\nu}(\omega) \\
& \geqslant\left[\sum_{\underline{i}=\left(i_{1}, i_{2}, \ldots, i_{n+K}\right)} \min _{\omega \in C_{\underline{i}} \cap Z^{\mathbb{N}}} r\left(g_{\omega}, n, \varepsilon\right)\right] \times \min _{\underline{i}} \mathbb{P}_{\nu}\left(C_{\underline{i}} \cap Z^{\mathbb{N}}\right) .
\end{aligned}
$$

As the image of the map $r(\cdot, n, \varepsilon): C_{\underline{i}} \rightarrow \mathbb{Z}_{+}$defined by $\omega \in C_{\underline{i}} \mapsto r\left(g_{\omega}, n, \varepsilon\right)$ is contained in the set of positive integers, it has a minimum in $\mathbb{Z}_{+}$, which guarantees that $\omega^{(i)}$ can be chosen as a point in $C_{\underline{i}}$ where the $\min _{B_{\underline{i}}} r\left(g_{\omega}, n, \varepsilon\right)$ is attained. This together with (4.4), the fact that $\mathbb{P}_{\nu}$ is a product measure and the homogeneity assumption on $\nu$ imply that

$$
\begin{aligned}
\int_{Y^{\mathbb{N}}} r\left(g_{\omega}, n, \varepsilon\right) d \mathbb{P}_{\nu}(\omega) & \geqslant\left[\sum_{\underline{i}=\left(i_{1}, i_{2}, \ldots, i_{n+K}\right)} \min _{\omega \in C_{\underline{i}} \cap Z^{\mathbb{N}}} r\left(g_{\omega}, n, \varepsilon\right)\right] \times \min _{\underline{i}} \mathbb{P}_{\nu}\left(C_{\underline{i}} \cap Z^{\mathbb{N}}\right) \\
& \geqslant \sum_{\underline{i}} r\left(g_{\omega(i)}, n, \varepsilon\right) \times \min _{\underline{i}} \prod_{j=0}^{n+K-1} \nu\left(B\left(p_{i_{j}}, \frac{\varepsilon}{4}\right) \cap Z\right) \\
& \geqslant r\left(\left.T_{G}\right|_{Z^{\mathbb{N}} \times X}, n, \varepsilon\right)\left(\frac{1}{L^{2}}\right)^{n+K}\left(\frac{1}{N_{Z}(\varepsilon)}\right)^{n+K}
\end{aligned}
$$


where $L>0$ is specified by the homogeneity of $\nu$ and does not depend on neither $\varepsilon$ nor $n$. Notice that the inequality

$$
\sum_{\underline{i}} r\left(g_{\omega^{(\underline{i})}}, n, \varepsilon\right) \geqslant r\left(\left.T_{G}\right|_{Z^{\mathbb{N}} \times X}, n, \varepsilon\right)
$$

is a consequence of the fact that, if $\left.\left\{x_{1}^{(i)}, \ldots, x_{r\left(g_{\omega}(i)\right.}, n, \varepsilon\right)\right\}$ is a $\left(g_{\omega^{(i)}}, n, \varepsilon\right)$-spanning set of $Z$ with smallest cardinality, then

$$
\bigcup_{\underline{i}}\left\{\left(\omega^{(\underline{i})}, x_{1}^{(\underline{i})}\right), \ldots,\left(\omega^{(\underline{i})}, x_{r\left(g_{\omega}^{(i)}, n, \varepsilon\right)}^{(i)}\right)\right\}
$$

is a $\left(\left.T_{G}\right|_{Z^{\mathbb{N}} \times X}, n, \varepsilon\right)$-spanning set. Besides, the inequality

$$
\min _{\underline{i}} \prod_{j=0}^{n+K-1} \nu\left(B\left(p_{i_{j}}, \frac{\varepsilon}{4}\right)\right) \geqslant\left(\frac{1}{L^{2}}\right)^{n+K}\left(\frac{1}{N_{Z}(\varepsilon)}\right)^{n+K}
$$

is due to the homogeneity of $\nu$, which implies that, for every $q \in \operatorname{supp} \nu$, any $p_{i_{j}}$ and all $\underline{i}$,

$$
\nu\left(B\left(p_{i_{j}}, \varepsilon\right)\right) \geqslant \frac{1}{L} \nu(B(q, \varepsilon)) \quad \forall \varepsilon>0
$$

and the fact that, as $\bigcup_{e \in E} B\left(e, \frac{\varepsilon}{4}\right)=Z$,

$$
1=\nu\left(\bigcup_{e \in E} B\left(e, \frac{\varepsilon}{4}\right)\right) \leqslant \sum_{e \in E} \nu\left(B\left(e, \frac{\varepsilon}{4}\right)\right) \leqslant N_{Z}(\varepsilon) L \nu\left(B\left(q, \frac{\varepsilon}{4}\right)\right)
$$

thus

$$
\nu\left(B\left(q, \frac{\varepsilon}{4}\right)\right) \geqslant \frac{1}{L} \frac{1}{N_{Z}(\varepsilon)}
$$

Therefore,

$$
\begin{aligned}
\overline{\operatorname{mim}}_{M}\left(X, \mathbb{S}, d, \mathbb{P}_{\nu}\right) & =\limsup _{\varepsilon \rightarrow 0^{+}} \frac{\limsup _{n \rightarrow \infty} \frac{1}{n} \log \int_{Z^{\mathbb{N}}} r\left(g_{\omega}, n, \varepsilon\right) d \mathbb{P}_{\nu}(\omega)}{-\log \varepsilon} \\
& \geqslant \overline{\operatorname{mdim}}_{M}\left(Z^{\mathbb{N}} \times X, T_{G}, D \times d\right)-\limsup _{\varepsilon \rightarrow 0^{+}} \frac{\log N_{Z}(\varepsilon)}{-\log \varepsilon} \\
& =\overline{\operatorname{mim}}_{M}\left(Z^{\mathbb{N}} \times X, T_{G}, D \times d\right)-\overline{\operatorname{dim}}_{B} Z \\
& =\overline{\operatorname{mim}}_{M}\left((\operatorname{supp} \nu)^{\mathbb{N}} \times X, T_{G}, D \times d\right)-\overline{\operatorname{dim}}_{B}(\operatorname{supp} \nu) .
\end{aligned}
$$

Consequently, if $\nu \in \mathcal{H}_{Y}$ and $\operatorname{supp} \nu=Y$, then Proposition 4.2 yields

$$
\overline{\operatorname{dim}}_{B}(Y)+\overline{\operatorname{mdim}}_{M}\left(X, \mathbb{S}, d, \mathbb{P}_{\nu}\right)=\overline{\operatorname{mdim}}_{M}\left(Y^{\mathbb{N}} \times X, T_{G}, D \times d\right)
$$

and the proof of Theorem A (b) is complete.

Remark 4.3. We observe that in the previous argument we only used a weaker version of the homogeneity of the measure, namely condition (2.7). 
Remark 4.4. We note that a priori the full support assumption in item (b) of Theorem A cannot be replaced by the weaker requirement of the existence of a sequence $\left(\nu_{n}\right)_{n \in \mathbb{N}}$ of probabilities in $\mathcal{H}_{Y}$ such that $d_{H}\left(\operatorname{supp} \nu_{n}, Y\right) \rightarrow 0$ as $n \rightarrow \infty$, where $d_{H}$ denotes the Hausdorff distance. Actually, for a $C^{0}$-generic homeomorphism $g$, the function $Z \mapsto \overline{\operatorname{mdim}}_{M}\left(X,\left.g\right|_{Z}, d\right)$ is not continuous when we endow the space of compact subsets in $\mathrm{X}$ with the Hausdorff metric (cf. [8]).

\section{Proof of Corollary II}

Assume that $\overline{\operatorname{dim}}_{B} Y<+\infty$ and there exists a Borel homogeneous probability measure $\nu_{0}$ on $Y$ with full support. Take $y \in Y$ and the Dirac measure $\delta_{y}$ supported on $y$, which is homogeneous. Therefore,

$$
\sup _{y \in Y}\left\{\overline{\operatorname{mdim}}_{M}\left(X, g_{y}, d\right)\right\} \leqslant \sup _{\nu \in \mathcal{H}_{Y}}\left\{\overline{\operatorname{dim}}_{B}(\operatorname{supp} \nu)+\overline{\operatorname{mdim}}_{M}\left(X, \mathbb{S}, d, \mathbb{P}_{\nu}\right)\right\}
$$

where, by Corollary I, one has

$$
\sup _{\nu \in \mathcal{H}_{Y}}\left\{\overline{\operatorname{dim}}_{B}(\operatorname{supp} \nu)+\overline{\operatorname{mdim}}_{M}\left(X, \mathbb{S}, d, \mathbb{P}_{\nu}\right)\right\}=\overline{\operatorname{mdim}}_{M}\left(Y^{\mathbb{N}} \times X, T_{G}, D \times d\right) .
$$

Moreover, by Theorem A (b) we know that, for every $\nu_{0} \in \mathcal{H}_{Y}$ such that $\operatorname{supp} \nu_{0}=Y$,

$$
\overline{\operatorname{mdim}}_{M}\left(Y^{\mathbb{N}} \times X, T_{G}, D \times d\right)=\overline{\operatorname{mdim}}_{M}\left(X, \mathbb{S}, d, \mathbb{P}_{\nu_{0}}\right)+\overline{\operatorname{dim}}_{B} Y .
$$

Therefore,

$$
\overline{\operatorname{mdim}}_{M}\left(X, \mathbb{S}, d, \mathbb{P}_{\nu_{0}}\right) \geqslant \sup _{y \in Y}\left\{\overline{\operatorname{mdim}}_{M}\left(X, g_{y}, d\right)\right\}-\overline{\operatorname{dim}}_{B} Y
$$

In particular, given $\eta \in \mathscr{P}(Y)$, we deduce that

$$
\overline{\operatorname{mdim}}_{M}\left(X, \mathbb{S}, d, \mathbb{P}_{\nu_{0}}\right) \geqslant \int_{Y} \overline{\operatorname{mdim}}_{M}\left(X, g_{y}, d\right) d \eta(y)-\overline{\operatorname{dim}}_{B} Y .
$$

In case $\overline{\operatorname{dim}}_{B} Y=0$ and $\eta=\nu_{0}$, we are reduced to

$$
\overline{\operatorname{midim}}_{M}\left(X, \mathbb{S}, d, \mathbb{P}_{\nu_{0}}\right) \geqslant \int_{Y} \overline{\operatorname{mdim}}_{M}\left(X, g_{y}, d\right) d \nu_{0}(y)
$$

Regarding the equality in (5.1), since $\overline{\operatorname{mdim}}_{M}\left(X, g_{y}, d\right) \leqslant \sup _{\alpha \in Y}\left\{\overline{\operatorname{mdim}}_{M}\left(X, g_{\alpha}, d\right)\right\}$ for every $y \in Y$ and

$$
\int_{Y} \overline{\operatorname{mdim}}_{M}\left(X, g_{y}, d\right) d \nu_{0}(y) \leqslant \sup _{y \in Y}\left\{\overline{\operatorname{mdim}}_{M}\left(X, g_{y}, d\right)\right\} \leqslant \overline{\operatorname{mdim}}_{M}\left(X, \mathbb{S}, d, \mathbb{P}_{\nu_{0}}\right)
$$

if we have an equality in (5.1) then $\overline{\operatorname{mim}}_{M}\left(X, g_{y}, d\right)=\sup _{\alpha \in Y}\left\{\overline{\operatorname{mdim}}_{M}\left(X, g_{\alpha}, d\right)\right\}$ for $\nu_{0}$ almost every $y \in Y$. 


\section{EXAMPLeS}

Example 6.1. Consider the set $Y=\left\{\frac{1}{n}: n \in \mathbb{N}\right\} \cup\{0\}$ endowed with the Euclidean distance $d$ in $\mathbb{R}$. It is known that $\overline{\operatorname{dim}}_{B} Y=\frac{1}{2}$ (cf. [1, p. 229]). If $\mu$ is a probability measure on $Y$ and $\delta>0$, we can find a finite set $Y_{\delta} \subset Y$ such that $\mu\left(Y_{\delta}\right) \geqslant 1-\delta$. This proves that $\overline{\operatorname{dim}}_{B} \mu=0$ (see Definition 2.5). In particular,

$$
\sup _{\mu} \overline{\operatorname{dim}}_{B} \mu<\overline{\operatorname{dim}}_{B} Y
$$

where the supremum is taken over all Borel probability measures $\mu$ on $Y$. Moreover, if $f:[0,1] \rightarrow[0,1]$ is a continuous map with positive upper metric mean dimension (whose existence is proved in $[8,20])$, then

$$
\overline{\operatorname{mdim}}_{M}\left(Y^{N} \times[0,1], \sigma \times f, D \times d\right)=\frac{1}{2}+\overline{\operatorname{mdim}}_{M}([0,1], f, d)
$$

while

$$
\overline{\operatorname{dim}}_{B} \mu+\overline{\operatorname{mdim}}_{M}\left([0,1], \mathbb{S}, d, \mathbb{P}_{\mu}\right)=\overline{\operatorname{mdim}}_{M}([0,1], f, d)
$$

for every probability $\mu$ on $Y$. This shows that, in general, one cannot replace $\overline{\operatorname{dim}}_{B}(\operatorname{supp} \nu)$ by $\operatorname{dim}_{B} \nu$ in Theorem A.

Example 6.2. Take $k \in \mathbb{N} \backslash\{1\}, Y=\{1,2, \ldots, k\}$ and $X=[0,1]$ with the Euclidean metric $d$, and consider a continuous map $g_{i}:[0,1] \rightarrow[0,1]$ for each $1 \leqslant i \leqslant k$ such that

$$
\overline{\operatorname{mdim}}_{M}\left([0,1], g_{i}, d\right) \leqslant \overline{\operatorname{mdim}}_{M}\left([0,1], g_{j}, d\right) \quad \forall i<j .
$$

We observe that every probability $\nu$ on $Y$ is homogeneous and satisfies

$$
\overline{\operatorname{dim}}_{B}(\operatorname{supp} \nu)=0=\overline{\operatorname{dim}}_{B} Y .
$$

Thus Theorem A informs that, for every full supported probability measure $\nu$ on $Y$,

$$
\overline{\operatorname{mdim}}_{M}\left([0,1], \mathbb{S}, d, \mathbb{P}_{\nu}\right)=\overline{\operatorname{mdim}}_{M}\left(Y^{\mathbb{N}} \times[0,1], T_{G}, D \times d\right) .
$$

Suppose now that $k=2$ and that

$$
\overline{\operatorname{mdim}}_{M}\left([0,1], g_{1}, d\right)<\overline{\operatorname{mdim}}_{M}\left([0,1], g_{2}, d\right) .
$$

Consider the measure $\delta_{1}$, which is homogeneous and maximizes the dimension (that is, $\left.\overline{\operatorname{dim}}_{B}(\operatorname{supp} \nu)=\overline{\operatorname{dim}}_{B} Y\right)$. Notice that

$$
\begin{aligned}
\overline{\operatorname{mdim}}_{M}\left([0,1], \mathbb{S}, d, \mathbb{P}_{\nu}\right) & =\overline{\operatorname{mdim}}_{M}\left([0,1], g_{1}, d\right)<\overline{\operatorname{mdim}}_{M}\left([0,1], g_{2}, d\right) \\
& \leqslant \overline{\operatorname{mdim}}_{M}\left(Y^{\mathbb{N}} \times[0,1], T_{G}, D \times d\right) .
\end{aligned}
$$

This shows that the full support requirement in item (b) of Theorem A cannot be replaced by a mere request on the full dimension of the support. In other words, although every full supported probability $\nu \in \mathcal{H}_{Y}$ attains the supremum in the variational principle stated in Theorem A, the same property may fail for dimension-maximizing measures. 
Example 6.3. Given $\alpha \in\left[\frac{1}{2}, 1\right]$, let $g_{\alpha}:[0,1] \rightarrow[0,1]$ be a continuous map such that $\overline{\operatorname{mdim}}_{M}\left([0,1], g_{\alpha}, d\right)=\alpha$, where $d$ is the Euclidean distance in $[0,1]$. The existence of these maps $g_{\alpha}$ was established in [8]. Take $Y=\left\{\frac{1}{n}: n \in \mathbb{N}\right\} \cup\{0\}$ and consider the continuous skew product

$$
\begin{aligned}
T_{G}: Y^{\mathbb{N}} \times[0,1] & \rightarrow Y^{\mathbb{N}} \times[0,1] \\
(\omega, x) & \mapsto\left(\sigma(\omega), f_{\omega}(x)\right)
\end{aligned}
$$

where

$$
f_{\omega}(x)= \begin{cases}x & \text { if } \omega \neq 111 \ldots \\ g_{\alpha}(x) & \text { otherwise. }\end{cases}
$$

6.3.1 Assume that $\nu=\delta_{1}$ (which does not have full support) and $\mathbb{P}_{\nu}=\nu^{\mathbb{N}}$. Such a $\nu$ is homogeneous, $\overline{\operatorname{dim}}_{B}(\operatorname{supp} \nu)=0$ and

$$
\overline{\operatorname{mdim}}_{M}\left([0,1], \mathbb{S}, d, \mathbb{P}_{\nu}\right)=\overline{\operatorname{mdim}}_{M}\left([0,1], g_{\alpha}, d\right)
$$

since the random walk $\mathbb{P}_{\nu}=\nu^{\mathbb{N}}$ only detects the dynamics of $g_{\alpha}$. Moreover,

$$
\overline{\operatorname{miim}}_{M}\left(Y^{\mathbb{N}} \times[0,1], T_{G}, D \times d\right)=\max \{\alpha, 1 / 2\}=\alpha
$$

and $\overline{\operatorname{dim}}_{B} Y=\frac{1}{2}$. Thus

$$
\overline{\operatorname{mdim}}_{M}\left([0,1], \mathbb{S}, d, \mathbb{P}_{\nu}\right)>\overline{\operatorname{mdim}}_{M}\left(Y^{\mathbb{N}} \times[0,1], T_{G}, D \times d\right)-\overline{\operatorname{dim}}_{B} Y .
$$

In particular,

$$
\sup _{\nu \in \mathcal{H}_{Y}} \overline{\operatorname{mdim}}_{M}\left(X, \mathbb{S}, d, \mathbb{P}_{\nu}\right)>\overline{\operatorname{mdim}}_{M}\left(Y^{\mathbb{N}} \times X, T_{G}, D \times d\right)-\overline{\operatorname{dim}}_{B} Y .
$$

6.3.2 Consider now $\eta=\sum_{n=1}^{\infty} \frac{1}{2^{n}} \delta_{\frac{1}{n}}$ and $\mathbb{P}_{\eta}=\eta^{\mathbb{N}}$. Observe that:

(i) $\overline{\operatorname{dim}}_{B}(\operatorname{supp} \eta)=\overline{\operatorname{dim}}_{B}(Y \backslash\{0\})=\frac{1}{2}$ since the upper box dimension is closure invariant.

(ii) $\eta$ has full support.

(ii) $\eta$ is not homogeneous: given $L>0$, if $y_{1}=\frac{1}{2}, y_{2}=0$ and $0<\varepsilon<\left(\frac{\log \left[1-(4 L)^{-1}\right]}{-\log 2}-1\right)^{-1}$,

$$
\eta\left(B\left(y_{1}, \varepsilon\right)\right)>L \eta\left(B\left(y_{2}, \varepsilon\right)\right) .
$$

If we choose $\alpha>\frac{1}{2}$, then

$$
\overline{\operatorname{mdim}}_{M}\left(Y^{\mathbb{N}} \times[0,1], T_{G}, D \times d\right)=\overline{\operatorname{mdim}}_{M}\left([0,1], g_{\alpha}, d\right)>\frac{1}{2}
$$

and

$$
\overline{\operatorname{mdim}}_{M}\left([0,1], \mathbb{S}, d, \mathbb{P}_{\eta}\right)=0
$$

So

$$
\overline{\operatorname{dim}}_{B}(\operatorname{supp} \eta)+\overline{\operatorname{mdim}}_{M}\left([0,1], \mathbb{S}, d, \mathbb{P}_{\eta}\right)<\overline{\operatorname{mdim}}_{M}\left(Y^{\mathbb{N}} \times[0,1], T_{G}, D \times d\right) .
$$

That is, the equality (3.1) in Theorem A fails, showing that the homogeneity assumption is essential on item (b) of Theorem A.

If, on the contrary, $\alpha=\frac{1}{2}$, then

$$
\overline{\operatorname{dim}}_{B}(\operatorname{supp} \eta)+\overline{\operatorname{mdim}}_{M}\left([0,1], \mathbb{S}, d, \mathbb{P}_{\eta}\right)=\overline{\operatorname{mdim}}_{M}\left(Y^{\mathbb{N}} \times[0,1], T_{G}, D \times d\right) .
$$

Thus (3.1) in Theorem A holds, even though $\eta$ is not homogeneous. 
Example 6.4. Consider the unit circle $S^{1}$ endowed with the Euclidean metric $d$ induced by the plane and take $Y_{1}=\{0,2\}$ and $Y_{2}=[0,1] \cup\{2\}$. Given continuous maps $f_{0}, f_{2}: S^{1} \rightarrow S^{1}$, let $\mathbb{S}_{1}$ denote the free semigroup action generated by $G=\left\{g_{\lambda}: \lambda \in\{0,2\}\right\}$, where $g_{\lambda}=f_{\lambda}$ for both $\lambda=0$ and $\lambda=2$, and let $\mathbb{S}_{2}$ stand for the free semigroup action generated by $H=\left\{h_{\lambda}: \lambda \in[0,1] \cup\{2\}\right\}$, where $h_{\lambda}=f_{0}$ if $\lambda \in[0,1]$ and $h_{2}=f_{2}$. If we consider a random walk $\mathbb{P}_{1}=\nu_{1}^{\mathbb{N}}$ and define $\mathbb{P}_{2}=\nu_{2}^{\mathbb{N}}$ with $\nu_{2}=\nu_{1}(\{0\}) \operatorname{Leb}_{[0,1]}+\nu_{1}(\{2\}) \delta_{2}$, then the two free semigroup actions $\left(\mathbb{S}_{1}, \mathbb{P}_{1}\right)$ and $\left(\mathbb{S}_{2}, \mathbb{P}_{2}\right)$ are isomorphic. Besides, it is easy to check that

$$
h\left(S^{1}, \mathbb{S}_{1}, \mathbb{P}_{1}, \varepsilon\right)=h\left(S^{1}, \mathbb{S}_{2}, \mathbb{P}_{2}, \varepsilon\right) \quad \forall \varepsilon>0 .
$$

So

$$
\overline{\operatorname{mdim}}_{M}\left(S^{1}, \mathbb{S}_{1}, d, \mathbb{P}_{1}\right)=\overline{\operatorname{mdim}}_{M}\left(S^{1}, \mathbb{S}_{2}, d, \mathbb{P}_{2}\right) .
$$

However, since these actions are driven by the shifts on the spaces $Y_{1}^{\mathbb{N}}$ and $Y_{2}^{\mathbb{N}}$ satisfying $\overline{\operatorname{dim}}_{B}\left(Y_{1}\right)=0$ and $\overline{\operatorname{dim}}_{B}\left(Y_{2}\right)=1$, then item (b) of Theorem A yields

$$
\overline{\operatorname{mdim}}_{M}\left(S^{1}, \mathbb{S}_{1}, d, \mathbb{P}_{1}\right)=\overline{\operatorname{mdim}}_{M}\left(Y_{1}^{\mathbb{N}} \times S^{1}, T_{G}, D_{1} \times d\right)
$$

and

$$
\overline{\operatorname{mdim}}_{M}\left(S^{1}, \mathbb{S}_{2}, d, \mathbb{P}_{2}\right)=\overline{\operatorname{mdim}}_{M}\left(Y_{2}^{\mathbb{N}} \times S^{1}, T_{H}, D_{2} \times d\right)-1 .
$$

Thus, the larger metric mean dimension of $T_{H}$ is compensated by $\overline{\operatorname{dim}}_{B}\left(Y_{2}\right)$. We remark that, although the metric mean dimension is metric dependent, in this locally constant setting we were able to change the metric and the probability measure in $Y_{2}$ (keeping the measure of the interval $[0,1]$ unchanged) while keeping the upper metric mean dimension of the semigroup action.

Example 6.5. Consider $Y=[0,1]$ and the unit circle $S^{1}$ with the multiplicative group structure induced by $\mathbb{C}$. Denote by $d$ the Euclidean metric in $S^{1}$.

6.5.1 Let $\mathbb{S}$ be the semigroup action generated by the family of rotations $\left(R_{\alpha}\right)_{\alpha \in[0,1]}$ on $S^{1}$ given by $z \in S^{1} \mapsto R_{\alpha}(z)=e^{2 \pi i \alpha} z$. Denote by $T_{G}$ the induced skew product on $[0,1]^{\mathbb{N}} \times S^{1}$ and take the random walk $\mathbb{P}_{\text {Leb }}=\operatorname{Leb}^{\mathbb{N}}$ in $[0,1]^{\mathbb{N}}$. Then Corollary IV indicates that

$$
\overline{\operatorname{mdim}}_{M}\left([0,1]^{\mathbb{N}} \times S^{1}, T_{G}, D \times d\right)=\overline{\operatorname{mdim}}_{M}\left(S^{1}, \mathbb{S}, d, \mathbb{P}_{\text {Leb }}\right)+1 .
$$

Moreover, for every $n \in \mathbb{N}, \omega \in[0,1]^{\mathbb{N}}$ and $\varepsilon>0$

$$
d\left(g_{\omega_{j} \cdots \omega_{1}}(x), g_{\omega_{j} \cdots \omega_{1}}(y)\right)=d(x, y) \quad \text { for every } 1 \leq j \leq n
$$

hence $r\left(g_{\omega_{n} \cdots \omega_{1}}, n, \varepsilon\right)=\left\lfloor\frac{1}{\varepsilon}\right\rfloor+1$. Consequently,

$$
h_{\text {top }}\left(\mathbb{S}, \mathbb{P}_{\text {Leb }}\right)=0=\overline{\operatorname{mdim}}_{M}\left(S^{1}, \mathbb{S}, d, \mathbb{P}_{\text {Leb }}\right) \text {. }
$$

Thus $\overline{\operatorname{mdim}}_{M}\left([0,1]^{\mathbb{N}} \times S^{1}, T_{G}, D \times d\right)=1$.

6.5.2 Consider now the semigroup action $\mathbb{S}$ generated by the family of endomorphisms $\left(g_{\alpha}\right)_{\alpha \in[0,1]}$ of $\left(S^{1}\right)^{\mathbb{N}}$ defined by

$$
g_{\alpha}\left(z_{1}, z_{2}, \cdots, z_{n}, \cdots\right)=\left(e^{2 \pi i \alpha} z_{1}, e^{2 \pi i \alpha} z_{2}, \cdots, e^{2 \pi i \alpha} z_{n}, \cdots\right) .
$$


If we take in $\left(S^{1}\right)^{\mathbb{N}}$ the metric

$$
(\underline{a}, \underline{b}) \quad \mapsto \quad \mathcal{D}(\underline{a}, \underline{b})=\sum_{i=1}^{\infty} \frac{d\left(a_{i}, b_{i}\right)}{2^{i}}
$$

then each $g_{\alpha}$ is an isometry as well. Denote by $T_{G}$ the induced skew product on $[0,1]^{\mathbb{N}} \times\left(S^{1}\right)^{\mathbb{N}}$ and take the random walk $\mathbb{P}_{\text {Leb }}=\operatorname{Leb}^{\mathbb{N}}$ in $[0,1]^{\mathbb{N}}$. Again by Corollary IV

$$
\overline{\operatorname{mdim}}_{M}\left([0,1]^{\mathbb{N}} \times\left(S^{1}\right)^{\mathbb{N}}, T_{G}, D \times \mathcal{D}\right)=\overline{\operatorname{mdim}}_{M}\left(\left(S^{1}\right)^{\mathbb{N}}, \mathbb{S}, \mathcal{D}, \mathbb{P}_{\text {Leb }}\right)+1 .
$$

Besides, as each $g_{\alpha}$ is an isometry, for every $n \in \mathbb{N}, \omega \in[0,1]^{\mathbb{N}}$ and $\varepsilon>0$ one has $r\left(g_{\omega_{n} \cdots \omega_{1}}, n, \varepsilon\right)=r\left(g_{\omega_{n} \cdots \omega_{1}}, 1, \varepsilon\right)$. This implies that

$$
\overline{\operatorname{mdim}}_{M}\left(\left(S^{1}\right)^{\mathbb{N}}, \mathbb{S}, \mathcal{D}, \mathbb{P}_{\text {Leb }}\right)=0
$$

and

$$
\overline{\operatorname{mdim}}_{M}\left([0,1]^{\mathbb{N}} \times\left(S^{1}\right)^{\mathbb{N}}, T_{G}, D \times \mathcal{D}\right)=1 .
$$

Remark. We can estimate the minimal cardinality of $(n, \varepsilon)$-spanning subset of $\left(S^{1}\right)^{\mathbb{N}}$ associated to each $g_{\omega_{n} \cdots \omega_{1}}$ as follows.

(a) Take the minimum $K \in \mathbb{N}$ such that $\sum_{i=1}^{K} \frac{1}{2^{i}}<\frac{\varepsilon}{2}$.

(b) Choose any point $p_{i} \in S^{1}$ for $i \geqslant K+1$.

(c) Since each $g_{\alpha}$ is an isometry, for $1 \leqslant i \leqslant K$, consider a minimal $\left(1, \frac{\varepsilon}{4}\right)$-spanning subset $\mathcal{R}_{i} \subset S^{1}$, whose cardinality is $\left\lfloor\frac{4}{\varepsilon}\right\rfloor+1$. Then, the set

$$
\mathcal{R}_{1} \times \mathcal{R}_{2} \times \cdots \mathcal{R}_{K} \times\left\{p_{K+1}\right\} \times\left\{p_{K+2}\right\} \cdots
$$

is an $(n, \varepsilon)$-spanning subset of $\left(S^{1}\right)^{\mathbb{N}}$. Thus

$$
r\left(g_{\omega_{n} \cdots \omega_{1}}, 1, \varepsilon\right) \leq\left(\left\lfloor\frac{4}{\varepsilon}\right\rfloor+1\right)^{K} .
$$

6.5.3 Let $T_{G}$ be the induced skew product on $[0,1]^{\mathbb{N}} \times\left(S^{1}\right)^{\mathbb{N}}$ associated to the free semigroup action $\mathbb{S}$ generated by the family of maps $\left(\widetilde{g}_{\alpha}\right)_{\alpha \in[0,1]}:\left(S^{1}\right)^{\mathbb{N}} \rightarrow\left(S^{1}\right)^{\mathbb{N}}$ defined by

$$
\widetilde{g}_{\alpha}\left(z_{1}, z_{2}, \cdots, z_{n}, \cdots\right)=\left(e^{2 \pi i \alpha} z_{2}, \cdots, e^{2 \pi i \alpha} z_{n}, \cdots\right) .
$$

Fix the random walk $\mathbb{P}_{\text {Leb }}=\operatorname{Leb}^{\mathbb{N}}$ in $[0,1]^{\mathbb{N}}$. Observe that $\widetilde{g}_{\alpha}=\sigma \circ g_{\alpha}$ for each $\alpha \in[0,1]$, where $g_{\alpha}$ is given by (6.1) and $\sigma$ denotes the shift map. Moreover, since $\sigma \circ g_{\alpha}=g_{\alpha} \circ \sigma$, then $\widetilde{g}_{\alpha}^{n}=\sigma^{n} \circ g_{\alpha}^{n}$ for every $n \geqslant 0$ and every $\alpha \in[0,1]$. If the metric $\mathcal{D}$ is defined as in (6.2) then the computation done to estimate the metric mean dimension of the shift ensures that

$$
s\left(\widetilde{g}_{\omega_{n} \cdots \omega_{1}}, 1, \varepsilon\right)=\left\lfloor\frac{1}{\varepsilon}\right\rfloor^{n} \quad \text { for every } n \text { and } \omega .
$$

Therefore, using Corollary IV, one obtains

$$
\overline{\operatorname{mdim}}_{M}\left([0,1]^{\mathbb{N}} \times\left(S^{1}\right)^{\mathbb{N}}, T_{G}, D \times \mathcal{D}\right)=\overline{\operatorname{mdim}}_{M}\left(\left(S^{1}\right)^{\mathbb{N}}, \mathbb{S}, \mathcal{D}, \mathbb{P}_{\text {Leb }}\right)+1=2 .
$$


Example 6.6. Consider $p \in \mathbb{N}$, a finite set of non-negative real numbers $\left\{\alpha_{i}: 1 \leqslant i \leqslant p\right\}$ such that $\alpha_{1} \neq 0$, and the free semigroup action $\mathbb{S}: G \times[0,1] \rightarrow[0,1]$ generated by

$$
G_{1}^{*}=\left\{g_{\alpha_{i}} \in C^{0}([0,1]): \overline{\operatorname{mim}}_{M}\left([0,1], g_{\alpha_{i}}, d\right)=\alpha_{i} \quad \forall 1 \leqslant i \leqslant p\right\} .
$$

The existence of such maps $g_{\alpha_{i}}$ was established in [8]. Take a Bernoulli probability measure $\mathbb{P}_{\nu}$ on $Y^{\mathbb{N}}=\{1, \ldots, p\}^{\mathbb{N}}$ determined by a probability vector $\left(a_{1}, \ldots, a_{p}\right)$. Set

$$
\alpha=\max \left\{\alpha_{i}: 1 \leqslant i \leqslant p\right\} .
$$

Without loss of generality we may assume that $\alpha=\alpha_{1}$. Afterwards, fix $\varepsilon>0$. For any $n \in \mathbb{N}$, let $C_{n}$ be the the cylinder of length $n$ which contains the sequence $\overline{1}=111 \ldots$. Then

$$
\int_{\{1, \ldots, p\}^{\mathbb{N}}} s\left(g_{\omega_{n}} \ldots g_{\omega_{1}}, n, \varepsilon\right) d \mathbb{P}_{\nu}(\omega) \geqslant \int_{C_{n}} s\left(g_{\omega_{n}} \ldots g_{\omega_{1}}, n, \varepsilon\right) d \mathbb{P}_{\nu}(\omega)=a_{1}^{n} s\left(g_{\alpha}, n, \varepsilon\right) .
$$

This implies that $h_{\mathrm{top}}\left(\mathbb{S}, \mathbb{P}_{\nu}, \varepsilon\right) \geqslant h_{\mathrm{top}}\left([0,1], g_{\alpha}, \varepsilon\right)$ for every $\varepsilon$. Consequently,

$$
\overline{\operatorname{mdim}}_{M}\left([0,1], \mathbb{S}, d, \mathbb{P}_{\nu}\right) \geqslant \alpha \text {. }
$$

On the other hand, as $\nu$ has full support, by Corollary III one gets

$$
\overline{\operatorname{mdim}}_{M}\left([0,1], \mathbb{S}, d, \mathbb{P}_{\nu}\right)=\overline{\operatorname{mdim}}_{M}\left(\{1, \ldots, p\}^{\mathbb{N}} \times[0,1], T_{G}, D \times d\right) .
$$

Since (cf. [20])

$$
\overline{\operatorname{mdim}}_{M}\left(\{1, \ldots, p\}^{\mathbb{N}} \times[0,1], T_{G}, D \times d\right) \leqslant \overline{\operatorname{dim}}_{B}\left(\{1, \ldots, p\}^{\mathbb{N}} \times[0,1]\right)=1
$$

we finally conclude that

$$
\alpha \leqslant \overline{\operatorname{mdim}}_{M}\left([0,1], \mathbb{S}, d, \mathbb{P}_{\nu}\right) \leqslant 1 .
$$

It is unclear to us whether there are examples where $\overline{\operatorname{mdim}}_{M}\left([0,1], \mathbb{S}, d, \mathbb{P}_{\nu}\right)$ can be strictly larger than $\alpha$.

Example 6.7. Let $f_{2}, f_{3}: \mathbb{S}^{1} \rightarrow \mathbb{S}^{1}$ be the doubling $\left(f_{2}(z)=z^{2}\right)$ and tripling $\left(f_{3}(z)=z^{3}\right)$ maps of the circle, respectively, and consider the push-forward dynamics $\left(f_{2}\right)_{*}$ and $\left(f_{3}\right)_{*}$ acting on the space $\mathscr{P}\left(\mathbb{S}^{1}\right)$ of Borel probability measures on the circle. By [11, Theorem 1.1], if $W_{p}$ denotes the $p$-Wasserstein metric in the space $\mathscr{P}\left(\mathbb{S}^{1}\right)$, then

$$
\overline{\operatorname{mdim}}_{M}\left(\mathscr{P}\left(\mathbb{S}^{1}\right),\left(f_{2}\right)_{*}, W_{p}\right) \geqslant p>0 \quad \text { and } \quad \overline{\operatorname{mdim}}_{M}\left(\mathscr{P}\left(\mathbb{S}^{1}\right),\left(f_{3}\right)_{*}, W_{p}\right) \geqslant 2 p>0 .
$$

Take $Y=\{1,2\}$. As a consequence of the discussion in Example 6.2, we deduce that, if $g_{1}=\left(f_{2}\right)_{*}, g_{2}=\left(f_{3}\right)_{*}$ and $\mathbb{S}$ is the free semigroup action generated by $\left\{g_{1}, g_{2}\right\}$, then

$$
\overline{\operatorname{miim}}_{M}\left(\mathscr{P}\left(\mathbb{S}^{1}\right), \mathbb{S}, W_{d}, \mathbb{P}_{\nu}\right) \geqslant \overline{\operatorname{mdim}}_{M}\left(\mathscr{P}\left(\mathbb{S}^{1}\right),\left(f_{3}\right)_{*}, W_{d}\right) \quad \forall \nu \in \mathscr{P}(Y) \backslash\left\{\delta_{1}\right\} .
$$

\section{REFERENCES}

[1] J. Bélair and S. Dubuc, Eds. Fractal Geometry and Analysis. NATO ASI Science Series C: 346, Springer Netherlands, 1991.

[2] A. Biś. An analogue of the variational principle for group and pseudogroup actions. Ann. Inst. Fourier 63:3 (2013) 839-863.

[3] C. Bonatti and L. J. Díaz and M. Viana. Dynamics beyond uniform hyperbolicity. (Encyclopaedia of Mathematical Sciences 102), Springer-Verlag Berlin Heidelberg, 2005.

[4] A. Bufetov. Topological entropy of free semigroup actions and skew-product transformations. J. Dynam. Control Systems 5 (1999) 137-143.

[5] M. Carvalho, F. Rodrigues, P. Varandas. Semigroups actions of expanding maps. J. Stat. Phys. 116:1 (2017) 114-136. 
[6] M. Carvalho, F. Rodrigues, P. Varandas. Quantitative recurrence for free semigroup actions. Nonlinearity $31: 3$ (2018) 864-886.

[7] M. Carvalho, F. Rodrigues and P. Varandas. A variational principle for free semigroup actions. Adv. Math. 334 (2018) 450-487.

[8] M. Carvalho, F. Rodrigues and P. Varandas. Generic homeomorphisms have full metric mean dimension Ergod. Th. \& Dynam. Sys. 2020, DOI:10.1017/etds.2020.130

[9] M. Gromov. Topological invariants of dynamical systems and spaces of holomorphic maps I. Math. Phys. Anal. Geom. 2:4 (1999) 323-415.

[10] K. Falconer. Fractal geometry: Mathematical foundations and applications. John Wiley \& Sons, Chichester, 1990.

[11] B. Kloeckner. Optimal transport and dynamics of expanding circle maps acting on measures. Ergodic Theory \& Dynam. Systems 33:2 (2013) 529-548.

[12] F. Ledrappier and P. Walters. A relativised variational principle for continuous transformations. J. Lond. Math. Soc. 16:3 (1977) 568-576.

[13] E. Lindenstrauss and B. Weiss. Mean topological dimension. Israel J. Math. 115 (2000) 1-24.

[14] E. Lindenstrauss and M. Tsukamoto. Double variational principle for mean dimension. Geom. Funct. Anal. Vol. 29 (2019) 1048-1109.

[15] Ya. Pesin. Dimension Theory in Dynamical Systems: Contemporary Views and Applications. (Lectures in Mathematics) University of Chicago Press, Chicago, 1997.

[16] F. Rodrigues, P. Varandas. Specification properties and thermodynamical properties of semigroup actions. J. Math. Phys. 57 (2016) 052704.

[17] D. Ruelle, On a compact with $\mathbb{Z}^{p}$-action satisfying expansiveness and specification. Trans. Amer. Math. Soc. 185 (1973) 237-251.

[18] M. Tsukamoto. Mean dimension of full shifts. Israel J. Math. 230:1 (2019) 183-193.

[19] M. Tsukamoto. Double variational principle for mean dimension with potential. Adv. Math. 361 (2020) 106935, 53 pp.

[20] A. Velozo, R. Velozo. Rate distortion theory, metric mean dimension and measure theoretic entropy. arXiv:1707.05762

CMup \& Departamento de Matemática, Universidade do Porto, Porto, Portugal.

E-mail address: mpcarval@fc.up.pt

Departamento de Matemática, Universidade federal do Rio Grande do Sul, Porto Alegre, BRAZIL.

E-mail address: fagnerbernardini@gmail.com

CMUP \& Departamento de Matemática e Estatística, Universidade Federal da Bahia, Bahia, BRAZIL.

E-mail address: paulo.varandas@ufba.br 